\title{
Haplotypes in the human Foxo1a and Foxo3a genes; impact on disease and mortality at old age
}

\author{
Maris Kuningas ${ }^{\star 1,2}$, Reedik Mägi ${ }^{3}$, Rudi GJ Westendorp ${ }^{1}$, P Eline Slagboom ${ }^{4}$, Maido Remm ${ }^{3}$ \\ and Diana van Heemst ${ }^{1}$ \\ ${ }^{1}$ Department of Gerontology and Geriatrics, Leiden University Medical Center, Leiden, The Netherlands; ${ }^{2}$ Department \\ of Biotechnology, Institute of Molecular and Cell Biology, University of Tartu, Tartu, Estonia; ${ }^{3}$ Department of \\ Bioinformatics, Institute of Molecular and Cell Biology, Tartu University, Tartu, Estonia; ${ }^{4}$ Section of Molecular \\ Epidemiology, Department of Medical Statistics, Leiden University Medical Center, Leiden, The Netherlands
}

Recently, the Daf-16 gene has been shown to regulate the lifespan of nematodes and flies. In mammals, the Daf-16 homologues are forkhead (FOXO) transcription factors, of which specific functions have been identified for Foxo1a and Foxo3a. Despite that, their influence on human age-related trajectories and lifespan is unknown. Here, we analysed the effect of genetic variance in Foxo1a and Foxo3a on metabolic profile, age-related diseases, fertility, fecundity and mortality. This study was carried out in the prospective population-based Leiden 85-plus Study, which includes 1245 participants, aged 85 years or more. The mean follow-up time was 4.4 years. Haplotype analyses of Foxo1a revealed that carriers of haplotype 3 'TCA' have higher HbA1c levels $(P=0.025)$ and a 1.14 -fold higher all-cause mortality risk $(P=0.021)$. This increase in mortality was attributable to death from diabetes, for which a 2.43-fold increase was observed $(P=0.025)$. The analyses with Foxo3a haplotypes revealed no differences in metabolic profile, fertility or fecundity. However, increased risks of stroke were observed for Foxo3a block-A haplotype 2 'GAGC' $(P=0.007)$ and haplotype 4 'AAAT' $(P=0.014)$ carriers. In addition, the haplotype 2 'GAGC' carriers had a 1.13-fold increased risk for all-cause mortality $(P=0.036)$ and 1.19-fold increased risk for cardiovascular mortality $(P=0.052)$. In conclusion, this study shows that genetic variation in evolutionarily conserved Foxo1a and Foxo3a genes influences lifespan in our study population.

European Journal of Human Genetics (2007) 15, 294-301. doi:10.1038/sj.ejhg.5201766; published online 24 January 2007

Keywords: Foxo1a; Foxo3a; mortality; lifespan; age-related disease; haplotype

Introduction

Insulin signalling has emerged as a conserved mechanism that influences the lifespan of several organisms. ${ }^{1,2}$ In Caenorhabditis elegans downregulation of the insulin/IGF-1 signalling (IIS) pathway activates Daf-16, and leads to increased lifespan. ${ }^{3,4}$ Among the genes regulated by Daf-16

*Correspondence: Maris Kuningas, Leiden University Medical Center (LUMC), Department of Gerontology and Geriatrics, C2-R, PO box 9600 , 2300 RC Leiden, The Netherlands.

Tel: + 3171526 6640; Fax: + 3171524 8159;

E-mail: M.Kuningas@lumc.nl

Received 25 July 2006; revised 20 November 2006; accepted 21 November 2006; published online 24 January 2007 are those implicated in glucose and lipid metabolism, fertility, stress response and defence mechanisms. ${ }^{5}$ In mammals, the main downstream targets of the IIS pathway are the forkhead box group $\mathrm{O}$ (FOXO) transcription factors, which are Daf-16 homologues. ${ }^{6}$ However, it remains to be elucidated whether FOXO proteins in mammals have a similar role as Daf-16 in C. elegans.

In mammals, the FOXO family consists of Foxo1a, Foxo3a, Foxo4 and Foxo6. These genes are expressed in all tissues albeit at varying degrees, suggesting that their physiological roles might be different. ${ }^{7-9}$ Distinct functions have been identified for Foxo1a and Foxo3a. Compared to other family members, Foxola seems to be 
the most important and functionally the most indispensable, as only the Foxo1a knockout mice were not viable. ${ }^{10,11}$ It has been shown that Foxo1a predominantly mediates the effects of insulin on metabolism, including its effects on hepatic glucose production. ${ }^{12}$ Mice overexpressing Foxo1a in liver and pancreatic $\beta$-cells have fasting hyperglycaemia and hepatic insulin resistance, leading to the development of diabetes in an agedependent manner. ${ }^{13-15}$ On the other hand, Foxo3a has been implicated in the suppression of follicular activation and thus in female fertility. ${ }^{11,16}$ These female Foxo3a knockout mice also displayed signs of premature ageing. Reduced lifespan in reproductively active females has been noted for a variety of species over the years. ${ }^{17}$ Hence, the phenotypes described above provide strong clues to the basic functions of Foxo1a and Foxo3a. Despite that, the role of FOXO proteins in humans has hardly been assessed. Recently, genetic variants in Foxo1a were associated with increased glucose levels and with a trend for early onset type-2 diabetes in a case-control study consisting of middle-aged participants. ${ }^{18}$ Nevertheless, the influence of Foxo1a and Foxo3a on human lifespan has not been assessed yet.

In this study, we analysed the effect of genetic variance in Foxo1a and Foxo3a on metabolic profile and mortality. In addition, associations with the prevalence of age-related diseases, fertility and fecundity were assessed. We used a haplotype-based approach, and the study was carried out in participants aged 85 years and older of the prospective population-based Leiden 85-plus Study.

\section{Materials and methods Study population}

The Leiden 85-plus Study is a prospective population-based study, in which inhabitants of Leiden, the Netherlands, aged 85 years or above, were invited to take part. There were no selection criteria related to health or demographic characteristics. The study population consists of two cohorts, cohort '87 and '97. Cohort '87 includes 977 participants aged 85 years and older, enrolled between 1987 and $1989 .{ }^{19}$ Cohort '97 consists of 599 subjects, all members of the 1912-1914 birth cohort, who were enrolled in the month of their 85th birthday between 1997 and $1999 .^{20}$ DNA was available for 682 participants from cohort ' 87 and for 563 people from cohort ' 97 . All the participants of the Leiden 85-plus Study were followed for mortality until 1st August 2005. Primary causes of death were obtained from the Dutch Central Bureau of Statistics and categorized according to the 10th International Classification of Diseases (ICD-10). The Medical Ethical Committee of the Leiden University Medical Center approved the study and informed consent was obtained from all the participants. We also genotyped 370 blood donors from Leiden and surrounding areas, ${ }^{21}$ to compare allele and haplotype frequencies between the elderly and the young.

Metabolic profile and BMI at baseline in cohort '97 HbA1c (haemoglobin A1c), triglycerides, C-reactive protein (CRP) and high-density lipoprotein (HDL)-cholesterol concentrations in serum were determined using fully automated analyzers (Hitachi 747 and 911; Hitachi, Ltd, Tokyo, Japan). Low-density lipoprotein (LDL)-cholesterol was estimated with the Friedewald equation. ${ }^{22}$ Body weight $(\mathrm{kg})$ and height $(\mathrm{cm})$ were measured in all participants and body mass index (BMI, $\mathrm{kg} / \mathrm{m}^{2}$ ) was calculated.

\section{Diabetes and cardiovascular pathologies at baseline in cohort '97}

Participants were classified as having diabetes when they met at least one of the following criteria: (1) history of diabetes obtained from the general practitioner or the subject's treating physician; (2) use of sulfonylureas, biguanides, or insulin, based on information obtained from the subject's pharmacist; or (3) nonfasting glucose of $\geq 11.1 \mathrm{mmol} / \mathrm{l}$. The prevalence of and the number of cardiovascular pathologies were obtained from the participants' general practitioners or nursing home physicians. In addition, electrocardiograms were recorded on a Siemens Siccard 440 and transmitted by telephone to the ECG Core Lab in Glasgow for automated Minnesota coding. ${ }^{23}$ Cardiovascular pathologies were classified as follows: myocardial infarction, myocardial ischemia, intermittent claudication, arterial surgery and stroke. ${ }^{24}$

\section{Fertility and fecundity in the combined cohort}

Birth dates of all the participants and their children, and the date(s) of marriage(s) were obtained from the registry of births, deaths and marriages of the municipality of Leiden and from the Central Bureau of Genealogy, the Netherlands. These participants were of childbearing age at a time of minimal fertility control for lack of modern contraceptive methods. Fertility and fecundity were assessed only in married female participants who were younger than 40 at the time of their marriage $(n=701)$. Women older than 40 at the time of their marriage were excluded from further analyses, owing to the rapid decline of fertility and fecundity that can be expected from that age onwards. Fertility was defined as having children or not. Fecundity was defined as the calculated time interval between the date of the first marriage and the birth date of the firstborn child. To minimize the selection of pregnancies conceived before marriage, women whose children were born before marriage or within the first 36 weeks (250 days) of marriage were excluded from analyses. ${ }^{25}$ 


\section{SNP selection and genotyping}

The single nucleotide polymorphisms (SNPs) from Foxo1a (GeneID 2308) and Foxo3a (GeneID 2309) were selected using the CEPH population (Utah residents with northern and western European ancestry) data from the International HapMap Project release no. $15 .^{26}$ All polymorphisms were genotyped with matrix-assisted laser desorption/ ionization time-of-flight (MALDI-TOF) mass spectrometry (MS), using the Sequenom MassARRAYtm methodology (Sequenom Inc, San Diego, CA, USA). Amplification reactions and parameters were based on the manufacturer's instructions.

\section{Statistical analysis}

The program Haploview ${ }^{27}$ was used to estimate the allele frequencies of the polymorphisms, test for HardyWeinberg equilibrium and estimate pair-wise linkage disequilibrium (LD) between the SNPs. Haplotypes and haplotype frequencies were calculated using SNPHAP software (http://www-gene.cimr.cam.ac.uk/clayton/software). Differences in allele and haplotype frequencies between the elderly and the young control group were tested using Fisher's exact test. The posterior probabilities of pairs of haplotypes per subject, as estimated by the SNPHAP, were used as weights in all the analyses. The haplotype analyses approach used in this study assumes an additive effect of the haplotypes, and details of this approach have been described elsewhere. ${ }^{28}$ Haplotypes with a frequency $<5 \%$ were combined and included in the following analyses, without reporting the results. Continuous variables were normally distributed except for HbA1c, triglycerides and CRP levels, which were therefore en-transformed.
Associations between haplotypes and metabolic profiles were analysed using a general linear model. Differences in the prevalence of cardiovascular pathologies, fertility and fecundity between haplotypes were tested, using binary logistic regression. All-cause and cause-specific mortality risks, with 95\% confidence intervals (CI), were calculated with Cox proportional hazard model, using left censoring to correct according to age for the delayed entry into the risk set. All analyses were sex adjusted and clustered by the individual identification number, to obtain robust standard errors. The common allele homozygote haplotype was used as the reference group. All the analyses were performed using STATA statistical software, version 9.0 (StataCorp LP, TX, USA).

\section{Results}

Using Hapmap data, we first examined the extent of LD in Foxo1a and Foxo3a. The polymorphisms of both genes were in LD (Figure 1a), which enabled us to select haplotypetagging SNPs (htSNPs) that would tag all haplotypes with frequencies $>1 \%$. From the Foxo1a gene three htSNPs that define one haplotype block, and from the Foxo3a gene nine htSNPs that define two haplotype blocks were chosen. In order to mark nonhaploblock regions, one SNP from Foxo1a and two SNPs from Foxo3a were selected (Figure 1a).

The 1245 participants of the Leiden 85-plus Study and 370 young blood-bank donors were genotyped for these polymorphisms. The genotype frequencies of the SNPs were in Hardy-Weinberg equilibrium and similar between the two elderly cohorts and the young control group (Table 1). As expected, all the htSNPs were in strong LD and
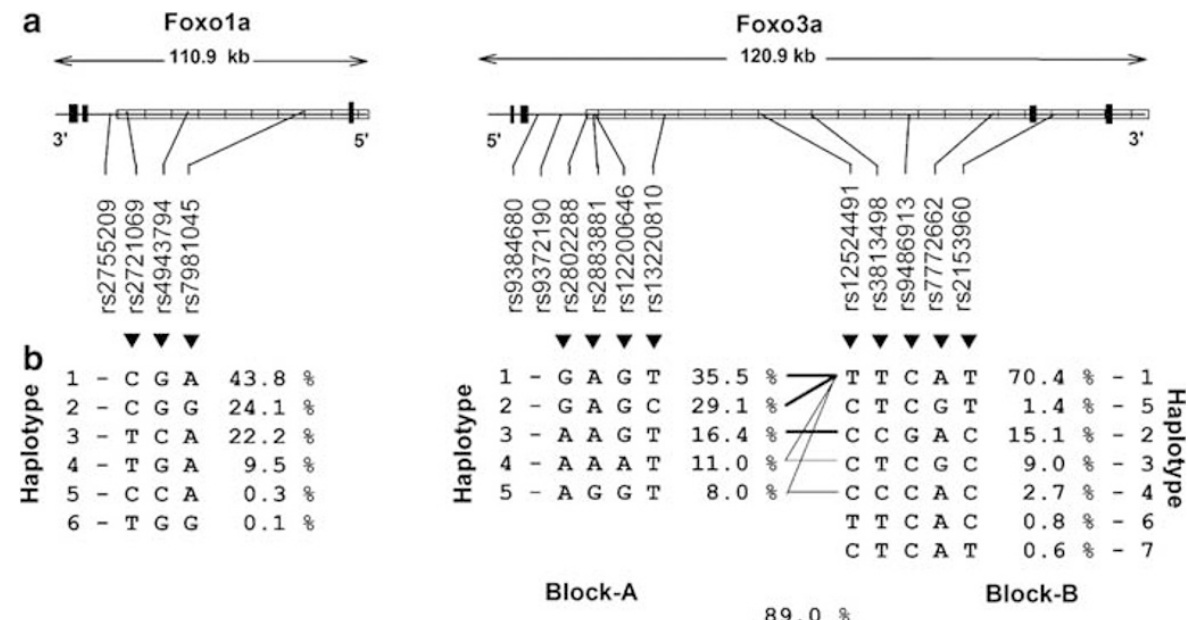

Figure 1 Foxo $1 a$ and Foxo3a gene structure, LD and haplotypes. (a) Genomic structure of Foxo1 $a$ and Foxo3a genes, where exons are represented by black boxes, and introns and intragenic regions by lines. The long striped horizontal box indicates the extent of LD based on the Hapmap data. Long vertical lines show the relative position of the SNPs analyzed in this study. (b) Haplotypes and their frequencies. For Foxo3a the lines between the block-A and block-B show the most common crossings from one block to the next, with thicker lines showing more common crossings than thinner lines. Beneath the crossing lines is shown the multilocus $\mathrm{D}^{\prime}$, which is a measure of the LD between the blocks. 
in Foxo1a gene they defined six haplotypes, of which four had frequencies $>5 \%$ and described $99.6 \%$ of the haplotype diversity (Figure 1b). In Foxo3a, the nine htSNPs defined two haplotype blocks. All five haplotypes in block$\mathrm{A}$ and three haplotypes in block-B had frequencies $>5 \%$ and described respectively $100 \%$ and $94.6 \%$ of haplotype diversity (Figure 1b). The overall and individual haplotype frequencies were not different between the elderly and young control group for neither of the genes (data not shown).

Table 1 Demographic characteristics of the study participants, and minor allele frequencies of the Foxo1a and Foxo3a polymorphisms

\begin{tabular}{|c|c|c|c|}
\hline & \multicolumn{2}{|c|}{ Leiden 85-plus Study } & \multirow[b]{2}{*}{ Young control } \\
\hline & Cohort '87 & Cohort '97 & \\
\hline Number & 682 & 563 & 370 \\
\hline Female $(n, \%)$ & $491(72 \%)$ & $375(67 \%)$ & $220(60 \%)$ \\
\hline Age (median, IQR) & $89(88-92)$ & $85(-)$ & $32(27-36)$ \\
\hline \multicolumn{4}{|l|}{ Foxo1 $a^{\mathrm{a}}$} \\
\hline rs2755209 (A/C) & 0.385 & 0.398 & 0.376 \\
\hline rs2721069 (C/T) & 0.321 & 0.310 & 0.292 \\
\hline rs4943794 (G/C) & 0.229 & 0.218 & 0.181 \\
\hline rs7981045 (A/G) & 0.229 & 0.263 & 0.260 \\
\hline \multicolumn{4}{|l|}{ Foxo3a $a^{\mathrm{a}}$} \\
\hline rs9384680 (T/G) & 0.029 & 0.039 & 0.036 \\
\hline rs9372190 (T/G) & 0.072 & 0.087 & 0.074 \\
\hline rs2802288 (G/A) & 0.365 & 0.341 & 0.354 \\
\hline rs2883881 (A/G) & 0.073 & 0.087 & 0.069 \\
\hline rs12200646 (G/A) & 0.113 & 0.109 & 0.128 \\
\hline rs13220810 (T/C) & 0.291 & 0.290 & 0.271 \\
\hline rs12524491 (T/C) & 0.300 & 0.270 & 0.290 \\
\hline rs3813498 (T/C) & 0.193 & 0.163 & 0.171 \\
\hline rs9486913 (C/G) & 0.166 & 0.136 & 0.142 \\
\hline rs7772662 (A/G) & 0.104 & 0.103 & 0.116 \\
\hline rs2153960 (T/C) & 0.293 & 0.262 & 0.281 \\
\hline
\end{tabular}

Abbreviation: IQR- interquartile range.

${ }^{\mathrm{a}}$ Minor allele frequencies. htSNPs are indicated in bold.
The data on metabolic profile, BMI, prevalence of diabetes and cardiovascular diseases were available for 563 participants of the cohort ' 97 . Haplotype analyses of the Foxo1a gene revealed that carriers of haplotype 3 'TCA' have $0.25 \mathrm{mmol} / \mathrm{l}$ higher HbA1c levels (95\% CI: 0.02-0.48, $P=0.025)$ compared with the levels in the carriers of the most common haplotype 1 'CGA' (Table 2). In addition, haplotype 3 'TCA' carriers had a trend for higher CRP levels and lower BMI (Table 2). Their risks for diabetes and myocardial infarction were also increased, although the association with diabetes was nonsignificant (OR 1.29, 95\% CI: $0.86-1.92, P=0.360)$ and the association with myocardial infarction just failed to reach significance (OR 1.41, 95\% CI: 1.00-2.00, $P=0.051$ ) (Supplementary Table 1). No differences in metabolic profile, diabetes or cardiovascular diseases were observed for any other Foxo1a haplotype.

Foxo1a haplotypes were also analysed for association with histories of fertility and fecundity in married women of the combined cohort $(n=701)$. These analyses revealed no associations with Foxo1a haplotypes (Supplementary Table 2).

To study the role of Foxo1a further, we assessed the association between Foxo1a haplotypes and mortality in 1245 participants of the combined cohort. During the mean follow-up time of 4.4 years, 1001 (80\%) of the participants had died. Of these deaths, 406 (41\%) were due to cardiovascular causes, $162(16 \%)$ were due to cancer and 431 (43\%) owing to other causes. Causes of death could not be obtained for two participants. Mortality analyses dependent on Foxo1a haplotypes revealed that carriers of haplotype 3 'TCA' had 1.14-fold increased all-cause mortality risks (95\% CI: $1.02-1.28, P=0.021)$ compared to the reference haplotype (Figure 2). This increase was not attributable to cardiovascular or cancer mortality, but to death from other causes (HR 1.28, 95\% CI: 1.09-1.51, $P=0.002)$. This category also included death due to diabetes $(n=14)$, for which an association with haplotype 3 'TCA' was observed (HR 2.43, 95\% CI: 1.12-5.27,

Table 2 Metabolic profile and BMI dependent on Foxo1a haplotypes in cohort '97 $(n=563)$

\begin{tabular}{|c|c|c|c|c|c|c|c|}
\hline & \multicolumn{7}{|c|}{ Foxo1a } \\
\hline & \multirow{2}{*}{$\begin{array}{c}\text { Haplotype } 1 \\
\text { Mean }(95 \% \mathrm{Cl})\end{array}$} & \multicolumn{2}{|c|}{ Haplotype 2} & \multicolumn{2}{|c|}{ Haplotype 3} & \multicolumn{2}{|c|}{ Haplotype 4} \\
\hline & & Difference $(95 \% \mathrm{Cl})^{\mathrm{a}}$ & P-value & Difference $(95 \% \mathrm{Cl})^{\mathrm{a}}$ & P-value & Difference $(95 \% \mathrm{CI})^{\mathrm{a}}$ & P-value ${ }^{a}$ \\
\hline Triglycerides $(\mathrm{mmol} / \mathrm{l})^{\mathrm{b}}$ & $1.57(1.44-1.69)$ & $+0.07(-0.04-0.17)$ & 0.593 & $+0.09(-0.03-0.21)$ & 0.152 & $+0.04(-0.12-0.19)$ & 0.816 \\
\hline $\mathrm{CRP}(\mathrm{mg} / \mathrm{l})^{\mathrm{b}}$ & $6.10(2.99-9.21)$ & $+0.88(-1.22-2.97)$ & 0.803 & $+1.90(-1.05-4.86)$ & 0.070 & $1.56(-2.34-5.46)$ & 0.346 \\
\hline $\mathrm{HDL}(\mathrm{mmol} / \mathrm{l})$ & $1.42(1.35-1.48)$ & $-0.01(-0.06-0.04)$ & 0.651 & $-0.04(-0.10-0.01)$ & 0.128 & $-0.06(-0.13-0.01)$ & 0.053 \\
\hline LDL (mmol/l) & $3.71(3.56-3.86)$ & $+0.12(-0.01-0.25)$ & 0.068 & $0.00(-0.13-0.14)$ & 0.971 & $+0.12(-0.08-0.31)$ & 0.245 \\
\hline
\end{tabular}

Abbreviations: BMI, body mass index; CRP, C-reactive protein; HbA1c, haemoglobin A1c; HDL, high-density lipoprotein cholesterol; LDL, low-density lipoprotein cholesterol.

*P-value $<0.05$.

aDifference compared to the most common haplotype 1.

${ }^{\mathrm{b}}$ Estimates presented for non-transformed and $P$-values for en-transformed data. 


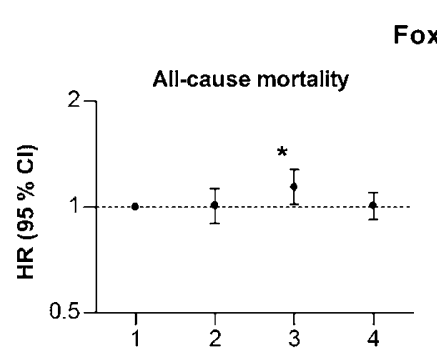

Foxo1a
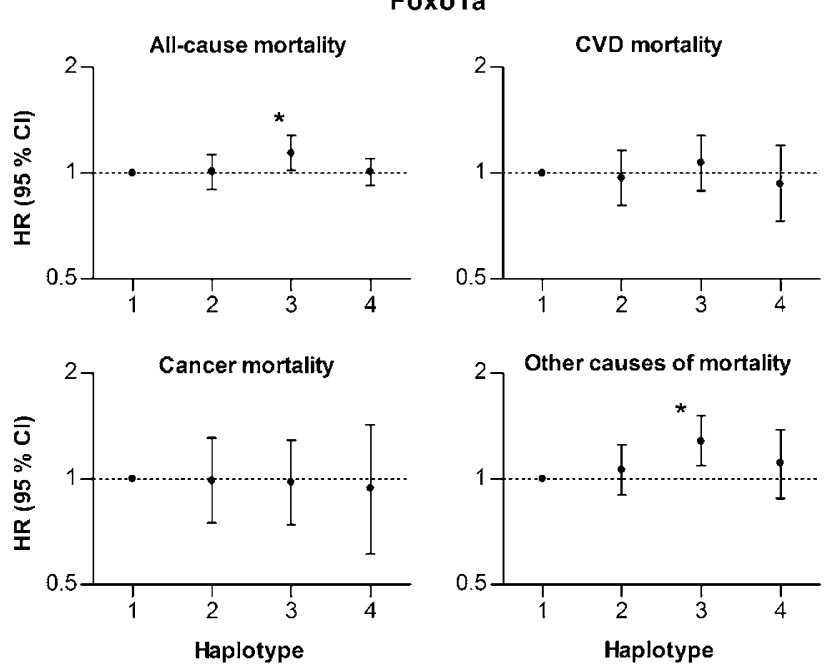

Figure 2 Foxo1a, all-cause and cause-specific mortality. Mortality risks were calculated in the combined cohort $(n=1245)$. Data are presented as hazard ratios (HR) with $95 \% \mathrm{Cl}$. The most common haplotype 1 was used as a reference group. ${ }^{*} P$-value $<0.05$ (see text).

$P=0.025)$. For the other Foxo1a haplotypes, no associations with all-cause or cause-specific mortalities were found (Figure 2).

The analyses with Foxo3a haplotypes revealed no differences in the various parameters of metabolic profile (Supplementary Table 3), fertility and fecundity (Supplementary Table 4). In contrast, increased risks of stroke for haplotype 2 'GAGC' (OR 1.92, 95\% CI: 1.19-3.08, $P=0.007$ ), and for haplotype 4 'AAAT' (OR 2.17, 95\% CI: 1.17-4.03, $P=0.014$ ) in Foxo3a block-A were observed (Table 3). In addition, the haplotype 2 'GAGC' carriers had 1.13-fold increased all-cause mortality (95\% CI; 1.01-1.26, $P=0.036)$ and 1.19 -fold increased cardiovascular mortality risks (HR 1.19, 95\% CI: 1.00-1.42, $P=0.052$ ) (Figure 3). There were no differences in cancer risk or in the risk from other causes of mortality in the Foxo3a haplotypes (Figure 3).

All the above-mentioned analyses were also performed with individual SNPs, which were selected to cover the nonhaploblock regions of the Foxo1a and Foxo3a genes. None of these polymorphisms were associated with any of the phenotypes analysed (data not shown).

\section{Discussion}

In this study, we report, for the first time, associations between haplotypes in the evolutionarily conserved Foxo1a and Foxo3a genes, and mortality in humans. For Foxo1a, haplotype 3 'TCA' was associated with higher HbA1c levels, with a trend for higher prevalence of diabetes and

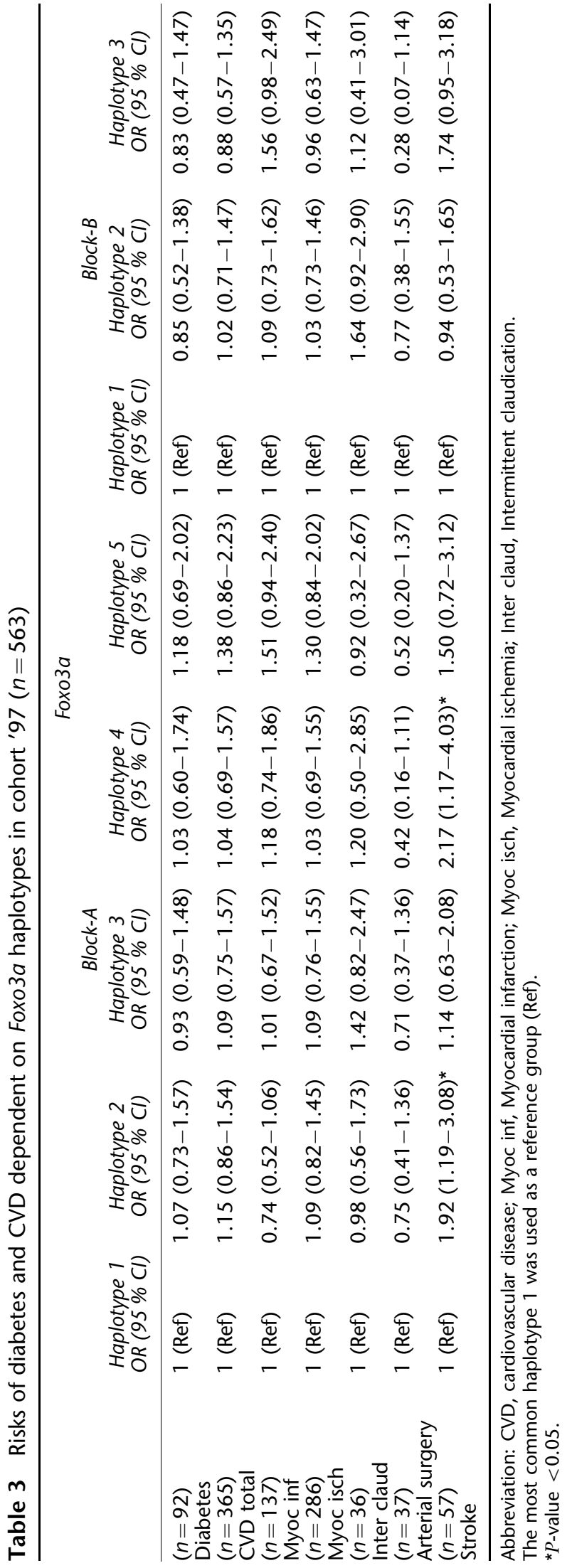



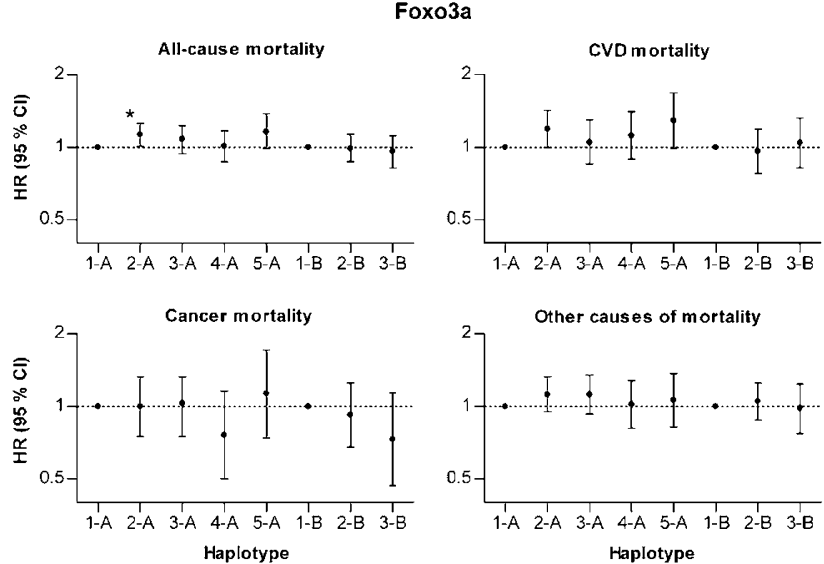

Figure 3 Foxo $3 a$, all-cause and cause-specific mortality. Mortality risks were calculated in the combined cohort $(n=1245)$. Data are presented as $\mathrm{HR}$ with $95 \% \mathrm{Cl}$. The most common haplotype 1 was used as a reference group. ${ }^{*} P$-value $<0.05$ (see text).

myocardial infarction, and increased mortality. Moreover, haplotype analyses of the Foxo3a gene revealed increased risks of stroke and mortality for haplotype 2 'GAGC' carriers.

FOXO transcription factors have emerged as candidate genes that are involved in lifespan regulation of various organisms. On the basis of the results from mouse models, it has been reasoned that Foxo1a influences mortality mainly by modifying the risks of diabetes. ${ }^{12}$ In this study, we observed an association between Foxo1a haplotype 3 'TCA' and HbA1c, which is the main risk factor for diabetes. In these haplotype carriers, the risks of diabetes and mortality were also increased. The observation that BMI was lower suggests that the susceptibility to diabetes in these elderly participants was not influenced by body composition. In principle, all diabetes at old age is due to type- 2 diabetes and is driven by insulin resistance and secondary exhaustion of the $\beta$-cell function. This implies that the Foxo1a transcription factors, which are normally downregulated by insulin signalling, are activated leading to increased transcription of Foxo1a target genes.

The role of Foxola in the development of diabetes has been previously assessed in a case-control study consisting of middle-aged participants. ${ }^{18}$ In that study, haplotypes in Foxo1a were associated with increased glucose levels, and with a trend for diabetes. In this study, we observed similar results, even though only one polymorphism was the same (rs2721069) between the studies. This difference in the analysed polymorphisms might explain the stronger associations observed in this study. However, on the basis of the evidence from both the studies, we conclude that, in humans, Foxo1a may influence glucose metabolism and contribute to the predisposition to diabetes, leading to increased mortality. In contrast, we found no evidence for the Foxo1a involvement in female fertility and fecundity, which were respectively defined as the ability to have children, and the probability to conceive within a specific period of time. $^{25}$

The other FOXO transcription factor, Foxo3a, has been implicated in a variety of biological processes, including metabolism, fertility, stress response and ageing. In this study, we found no associations between Foxo3a haplotypes, human fertility and fecundity. In mouse models, the lack of Foxo3a resulted in an age-dependent decline of fertility in homozygous knockout mice, whereas heterozygous mice were indistinguishable from the wild types. ${ }^{11}$ This suggests that mutations or severe disruptions of human Foxo3a might lead to phenotypes similar to those observed in mice. Similar to the results with fertility and fecundity, we found no association with metabolic profile and Foxo3a haplotypes. Despite that, carriers of haplotype 2 'GAGC' in Foxo3a block-A, had increased risks of stroke, and increased mortality, which was partly attributable to increased cardiovascular mortality. The mechanisms through which Foxo3a influences the occurrence of stroke are unknown, but the involvement of Foxo3a in the mediation of oxidative stress responses ${ }^{29}$ might be a possibility.

Several studies have implicated Foxo1a and Foxo3a in the development of tumours. ${ }^{30,31}$ In addition, FOXO proteins have been shown to induce cell-cycle arrest, DNA repair and apoptosis, thereby making them attractive candidates for tumour suppression. The results of this study did not reveal any significant differences in the estimates of cancer mortality risk for the different Foxo1a and Foxo3a haplotypes. For Foxo1a, we expected opposite results, as predisposition to diabetes and protection against cancer have been associated with Foxo gain of function. ${ }^{32,33}$

The regulation of an organism's lifespan is complex and depends not only on multiple genetic, epigenetic and environmental factors, but also on the interaction between them. In this study, we used a candidate gene approach, which relies on predicting the identity of the correct gene or genes on the basis of biological hypothesis, or the location of the candidate within a previously determined region of linkage. This approach, however, will identify only a fraction of the genetic factors that contribute to the complex phenotype. A complementary approach would be a whole genome-association study that surveys most of the genome for causal genetic variants. Such an approach could reveal valuable additional information on the genetic bases of human lifespan regulation.

The first strength of this study is the haplotype-tagging SNP approach, which most probably captured the common genetic variations present in both the genes. The Foxo1a haplotype 3 'TCA' consists of intronic SNPs. This suggests that these SNPs might be in LD with a nearby functional polymorphism that drives the observed associations. Since the LD in the Foxo1a gene extends beyond $5^{\prime}-\mathrm{UTR}$, then 
the functional SNP hypothesized probably is located in the regulatory region. Therefore, in addition to replication, further studies are needed to pinpoint the location of the functional variant and to prove its influence on the Foxo1a function. Other strengths of the study were the possibility of estimating several intermediate phenotypes in one cohort, and the prospective analyses. The high prevalence of age-associated diseases and mortality in this cohort excludes the possibility that this cohort consists of healthy survivors only. A limitation of the study concerns the reproductive data, which were acquired from registries; therefore, all conception times and fecundity rates were calculated. In addition, taking into account the number of tests performed, adjustment for multiple testing would eliminate all the significant $P$-values observed.

In conclusion, the present study shows that human homologues of genes identified as influencing the lifespans of model organisms, have the same impact in humans. In this study, we observed biologically plausible influences of Foxo1a and Foxo3a haplotypes on age-related trajectories and mortality.

\section{Acknowledgements}

This work was supported by an Innovative Orientated Research (IOP) grant from the Dutch Ministry of Economic Affairs (Grant no. IGE010114), and by the Centre for Medical Systems Biology (CMSB), which is a centre of excellence approved by the Netherlands Genomics Initiative/Netherlands Organization for Scientific Research (NWO). MK was supported by a Marie Curie Fellowship of the European Community program EUROGENDIS 'The Genetic Basis of Disease' (contract no. QLGA-GH-00-60005-59). RM and MR were supported by the Estonian Ministry of Education and Research (Grant no. 0182649s04). All authors have seen and agreed with the contents of the manuscript, and none of the authors has any potential conflict of interest to disclose. The authors would like to thank A M de Craen, JJ Houwing-Duistermaat and $H-W$ Uh for the help in data analyses.

\section{References}

1 Guarente L, Kenyon C: Genetic pathways that regulate ageing in model organisms. Nature 2000; 408: 255-262.

2 Tatar M, Bartke A, Antebi A: The endocrine regulation of aging by insulin-like signals. Science 2003; 299: 1346-1351.

3 Tissenbaum HA, Ruvkun G: An insulin-like signaling pathway affects both longevity and reproduction in Caenorhabditis elegans. Genetics 1998; 148: 703-717.

4 Ogg S, Paradis S, Gottlieb S et al: The Fork head transcription factor DAF-16 transduces insulin-like metabolic and longevity signals in C. Elegans. Nat 1997; 389: 994-999.

5 Murphy CT, McCarroll SA, Bargmann CI et al: Genes that act downstream of DAF-16 to influence the lifespan of Caenorhabditis elegans. Nature 2003; 424: 277-283.

6 Lin K, Dorman JB, Rodan A, Kenyon C: daf-16: An HNF-3/ forkhead family member that can function to double the life-span of Caenorhabditis elegans. Science 1997; 278: 1319-1322.

7 Anderson MJ, Viars CS, Czekay S, Cavenee WK, Arden KC: Cloning and characterization of three human forkhead genes that comprise an FKHR-like gene subfamily. Genomics 1998; 47: $187-199$.

8 Biggs WHI, Cavenee WK, Arden KC: Identification and characterization of members of the FKHR (FOX O) subclass of winged- helix transcription factors in the mouse. Mamm Genome 2001; 12 : $416-425$.

9 Furuyama T, Nakazawa T, Nakano I, Mori N: Identification of the differential distribution patterns of mRNAs and consensus binding sequences for mouse DAF-16 homologues. Biochem J 2000; 349: 629-634.

10 Furuyama T, Kitayama K, Shimoda Y et al: Abnormal angiogenesis in Foxo1 (Fkhr)-deficient mice. J Biol Chem 2004; 279: 3474134749 .

11 Hosaka T, Biggs WH, Tieu D et al: Disruption of forkhead transcription factor (FOXO) family members in mice reveals their functional diversification. Proc Natl Acad Sci U S A 2004; 101: $2975-2980$.

12 Barthel A, Schmoll D, Unterman TG: FoxO proteins in insulin action and metabolism. Trends Endocrinol Metab 2005; 16: $183-189$.

13 Altomonte J, Richter A, Harbaran S et al: Inhibition of Foxo1 function is associated with improved fasting glycemia in diabetic mice. Am J Physiol Endocrinol Metab 2003; 285: E718-E728.

14 Nakae J, Biggs WH, III, Kitamura T et al: Regulation of insulin action and pancreatic beta-cell function by mutated alleles of the gene encoding forkhead transcription factor Foxo1. Nat Genet 2002; 32: $245-253$

15 Zhang W, Patil S, Chauhan B et al: FoxO1 regulates multiple metabolic pathways in the liver: effects on gluconeogenic, glycolytic, and lipogenic gene expression. I Biol Chem 2006; 281: $10105-10117$.

16 Castrillon DH, Miao L, Kollipara R, Horner JW, DePinho RA: Suppression of ovarian follicle activation in mice by the transcription factor Foxo3a. Science 2003; 301: 215-218.

17 Partridge L, Gems D, Withers DJ: Sex and death: what is the connection? Cell 2005; 120: 461-472.

18 Karim MA, Craig RL, Wang X, Hale TC, Elbein SC: Analysis of FOXO1A as a candidate gene for type 2 diabetes. Mol Genet Metab 2006; 88: 171-177.

19 Weverling-Rijnsburger AWE, Blauw GJ, Lagaay AM, Knook DL, Meinders AE, Westendorp RGJ: Total cholesterol and risk of mortality in the oldest old. The Lancet 1997; 350: 1119-1123.

20 der Wiel AB, van Exel E, de Craen AJM et al: A high response is not essential to prevent selection bias: results from the Leiden 85-plus study. J Clin Epidemiol 2002; 55: 1119-1125.

21 Heijmans BT, Gussekloo J, Kluft C et al: Mortality risk in men is associated with a common mutation in the methylene-tetrahydrofolate reductase gene (MTHFR). Eur J Hum Genet 1999; 7: 197-204.

22 Friedewald WT, Levy RI, Fredrickson DS: Estimation of the concentration of low-density lipoprotein cholesterol in plasma, without use of the preparative ultracentrifuge. Clin Chem 1972; 18: 499-502.

23 Macfarlane PW, Latif S: Automated serial ECG comparison based on the Minnesota code. J Electrocardiol 1996; 29 (suppl.): $29-34$.

24 van Exel E, Gussekloo J, Houx P et al: Atherosclerosis and cognitive impairment are linked in the elderly. The Leiden $85-$ plus Study. Atherosclerosis 2002; 165: 353-359.

25 van Dunne FM, De Craen AJ, Heijmans BT, Helmerhorst FM, Westendorp RG: Gender-specific association of the factor $\mathrm{V}$ Leiden mutation with fertility and fecundity in a historic cohort. The Leiden 85-Plus Study. Hum Reprod 2006; 21: 967-971.

26 The International HapMap Consortium: A haplotype map of the human genome. Nature 2005; 437: 1299-1320.

27 Barrett JC, Fry B, Maller J, Daly MJ: Haploview: analysis and visualization of LD and haplotype maps. Bioinformatics 2005; 21: 263-265.

28 Wallenstein S, Hodge SE, Weston A: Logistic regression model for analyzing extended haplotype data. Genet Epidemiol 1998; 15: $173-181$.

29 Lehtinen MK, Yuan Z, Boag PR et al: A conserved MST-FOXO signaling pathway mediates oxidative-stress responses and extends life span. Cell 2006; 125: 987-1001. 
30 Galili N, Davis RJ, Fredericks WJ et al: Fusion of a fork head domain gene to PAX3 in the solid tumour alveolar rhabdomyosarcoma. Nat Genet 1993; 5: 230-235.

31 Hillion J, Le Coniat M, Jonveaux P, Berger R, Bernard OA: AF6q21, a novel partner of the MLL gene in $\mathrm{t}(6 ; 11)(\mathrm{q} 21 ; \mathrm{q} 23)$, defines a forkhead transcriptional factor subfamily. Blood 1997; 90: 37143719.
32 Yang $\mathrm{H}$, Zhao $\mathrm{R}$, Yang HY, Lee MH: Constitutively active FOXO4 inhibits Akt activity, regulates p27 Kip1 stability, and suppresses HER2-mediated tumorigenicity. Oncogene 2005; 24 : $1924-1935$.

$33 \mathrm{Hu} \mathrm{MC}$, Lee DF, Xia W et al: IkappaB kinase promotes tumorigenesis through inhibition of forkhead FOXO3a. Cell 2004; 117: 225-237.

Supplementary Information accompanies the paper on European Journal of Human Genetics website (http://www.nature.com/ejhg) 